\title{
Isobath variation and trapping of continental shelf waves
}

\author{
G. KA O ULLAS $\dagger$, AND E. R. JOHNSON \\ Department of Mathematics, University College London, Gower Street, London WC1E 6BT, \\ UK
}

(Received 6 February 2012)

Since Trösch (1986) found trapped sub-inertial oscillations in computations of low frequency variability in the Lake of Lugano, models of trapping have generally considered evenly spaced isobaths parallel to shorelines with approximate boundary conditions at any shelf-ocean boundary. Here an asymptotic analysis for slowly-varying topography and accurate spectral computations demonstrate trapping on non-rectilinear shelves. It is shown that changes in any of three factors, isobath curvature, distance from the coast and the shelf-break, and the slope at the shelf-break, are sufficient on their own to give trapping. Continental shelves that abut smoothly onto the open ocean are considered thus avoiding the shelf-ocean boundary condition approximation and allowing the accuracy of previous approximations to be assessed.

\section{Introduction}

Trapped modes are known to exist in a number of physical settings. Existence theorems have been proved rigorously for the acoustic (see Evans et al. 1994; Aslanyan et al. 2000; Davies and Parnovski 1998) and the quantum (Exner and Seba 1989; Duclos and Exner 1995; Dittrich and Kriz 2002; Krejčiř́ík and Kříž 2005) problems and asymptotic and numerical results have been obtained for problems in elasticity (Gridin et al. 2005; Kaplunov et al. 2005). Trapped modes are also known to exist along continental shelves, in particular when continental shelf waves (CSWs) are trapped.

CSWs are low frequency disturbances of ocean currents that occur near coastlines over the continental shelf. They are considered as a type of planetary wave having a period of a few days and exist due to the presence of rotation and depth changes. Since they were first discovered by Hamon $(1962,1963)$ and Robinson $(1964)$, most theoretical results are concerned with shelf waves that propagate along continental shelves with unchanged offshore profile but decay away from the coast. There is far less discussion in the literature regarding geographically localised regions of wave motion where modes decay both away from the coast and along the shelf, away from the wave region. Such a behaviour is possible when sufficient changes in the shelf geometry are present, since at a fixed frequency a given mode can change from propagating to decaying. These waves will be referred to as trapped CSWs (tCSWs).

The first results showing the existence of tCSWs were presented in the papers by Stocker and Hutter $(1986,1987)$ in a rectangular lake with idealised topography and as the low-frequency modes found in a finite-element model of Lake Lugano by Trösch (1986). Stocker and Johnson $(1989,1991)$ presented simplified quasi-analytical models for a semi-infinite channel with terminating bay zone and for an estuary mouth on a 
coastal shelf, respectively, which admit tCSWs. Straight, rectilinear shelves (i.e. where the depth changes uniformly away from the coast) do not admit trapped modes and in the simplest case of CSWs, making the non-divergent, barotropic approximation, the topographic Rossby wave equation is invariant under conformal mappings, hence any geometry that can be mapped conformally to a (straight) rectilinear shelf cannot support trapped modes. Questions still remained as to whether tCSWs can exist in other (smooth) geometries e.g. in bays or headlands and the effect of alongshore variations in the isobaths.

One of these questions was answered in Johnson et al. (2006) (JLP, here) where spectral theory is used to prove that under relatively weak (geometric) assumptions, tCSWs can exist on a rectilinear shelf lying between a smooth curved coastline and a constantdepth open ocean. The shelf is modelled as a strip taking Dirichlet boundary condition at the coast and Neumann boundary condition at the shelf-ocean boundary and if the waveguide is bent towards the Dirichlet condition (i.e. towards shallow water) a trapped mode exists. Considering a similar geometric model to the one in JLP, Postnova and Craster (2008) and Johnson et al. (2011) show, using an asymptotic scheme, that tCSWs can exist in a curved coastline, which is straight asymptotically (i.e. the curvature goes to zero at infinity) a weaker condition than the one in JLP, where the curvature has compact support. Also, in Kaoullas and Johnson (2010b) trapped modes are constructed without restricting attention to weak curvature and thus the behaviour of the solutions is examined when the curvature exceeds the upper bound of JLP. These three methods rely heavily on the Neumann boundary condition approximation, at the shelf-ocean boundary, which is valid only in the long wave limit.

The purpose of this paper is to extend previous results and show the existence of tCSWs along a curved shelf abutting a flat open ocean. In addition we present analogous results for straight shelves with varying bathymetry both across and along the coast. In Section 2 we present the governing equation and then employ its asymptotic expansion by modelling the shelf as a waveguide. Then, we discuss the existence of trapping when a straight shelf is considered with nonrectilinear depth profiles (i.e. we allow for depth changes along the coastline as well). In particular, we investigate the existence of trapped modes in the cases of a submerged ridge and of a submerged valley (for an oceanic waveguide). Assuming that the scale of the along-shelf variations of the isobaths is large compared to the shelf width (which implies that the wavelength in the alongshelf direction is much larger than in the cross-shelf direction), we reduce our problem to finding (analytical) solutions of a second order ordinary differential equation in the along-shelf direction. It is shown that in this particular case of a submerged ridge, when the depth is perturbed slightly, a trapped mode always exists, while in the case of a submerged valley, trapping is impossible. The full equations including a semi-infinite open ocean region, thus removing the Neumann shelf-ocean approximate boundary condition, are solved with an accurate spectral method. The numerical results are in close agreement with the asymptotic results and also allow consideration of the existence of trapped modes along a shelf with alongshore depth variations of the same order as the shelf width abutting an open ocean and assessing the accuracy of the Neumann shelf-ocean boundary condition. The simple physical mechanism behind the localisation is also described here: modes are trapped in regions where the local propagation frequency is higher than maximum possible frequency for propagating modes in the far-field. Curving the domain with shallower fluid on the inside of the curve, increasing the displacement of the shelf-break from the coast, or shelf-steepening, each individually or in combination raise the local propagation frequency on shelves that do no vary along the coast. Thus if these changes are localised then trapped modes should be possible.

Such trapped modes are constructed in Section 3. A curved coast is modelled as in 
JLP, Postnova and Craster (2008), Kaoullas and Johnson (2010b) and Johnson et al. (2011) who show that trapped modes can exist when a coast curves to form a headland. To assess the second factor a straight shelf that admits no trapped modes is constructed in section 3.2. A flat bay (of finite extent) is then added to the coast, which increases the distance of the shelf-break from the coast, similarly to Johnson and Kaoullas (2011) and thus raises the local cutoff frequency of waves (compared to the cutoff frequency in the farfield which remains unchanged) and hence allows trapped modes. To assess the third factor a straight shelf is constructed where the slope of the shelf-break is changes locally while the distance of the shelf-break from the wall remains constant. It is shown that a steepening shelf break leads to trapping.

\section{Non rectilinear topographies along straight coastlines}

\subsection{Governing Equation}

CSWs are well described within the barotropic, rigid-lid approximation (Buchwald and Adams (1968); LeBlond and Mysak (1978); JLP) by the topographic Rossby wave equation:

$$
\begin{array}{r}
\nabla \cdot\left(H^{-1} \nabla \Psi_{t}\right)+2 \Omega \hat{\mathbf{z}} \cdot\left(\nabla \Psi \times \nabla H^{-1}\right)=0, \\
\Psi=0, \quad \text { at the coast, } \\
\nabla \Psi \rightarrow 0, \quad \text { in the open ocean. }
\end{array}
$$

Here, $\Psi(x, y, t)$ is the mass transport stream function, $H(x, y)$ the local depth, $\hat{\mathbf{z}}$ a vertical unit vector, $\nabla$ the horizontal gradient operator and $\Omega$ is the vertical component of the Earth's rotation. The coastline is considered to be impermeable giving the Dirichlet boundary condition (2.2) that the streamfunction vanishes along the coast. Additionally, the depth becomes constant in the open ocean region forcing the velocity associated with CSWs to vanish far from the coast giving the boundary condition (2.3).

\subsection{Asymptotic method}

In this section we examine the existence of trapped modes in a straight coastline with depth variations both along and across the shore. Assuming that the scale of the alongshelf variations of the isobaths is large compared to the shelf width (which implies that the wavelength in the along-shelf direction is much larger than in the cross-shelf direction), we reduce our problem to finding (analytical) solutions of a second order ordinary differential equation in the along-shelf direction.

Consider a straight shelf, modelled as a waveguide, with depth variations both along and across the shore (see figure 1). If the coastline is straight and the depth profile is rectilinear, the boundary condition at the shelf-ocean boundary has a simple form (e.g. Buchwald and Adams 1968; Kaoullas and Johnson 2010a). However, for nonrectilinear geometries, where either the coast or the depth profile or both are not functions of a single coordinate, the boundary condition becomes an integral equation which leads to complicated expressions.

It is common in the literature (e.g. JLP, Postnova and Craster (2008), Kaoullas and Johnson (2010b), Johnson et al. (2011)), in such cases to replace (2.3) with a Neumann boundary condition

$$
\hat{\mathbf{n}} \cdot \nabla \Psi=0,
$$

where $\hat{\mathbf{n}}$ is normal to the shelf ocean boundary. Condition (2.4) is an approximation to (2.3), valid for waves sufficiently long compared to the shelf width, but is essential for the asymptotic analysis which follows. 


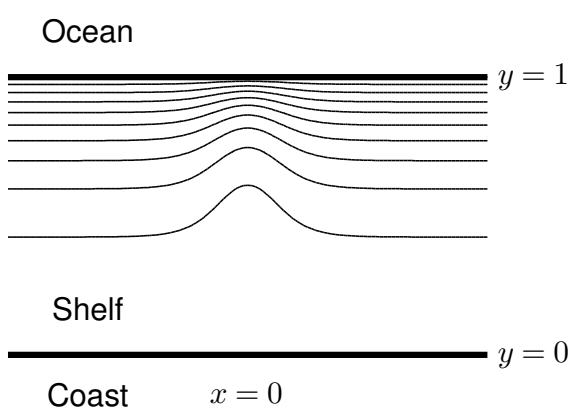

(a)

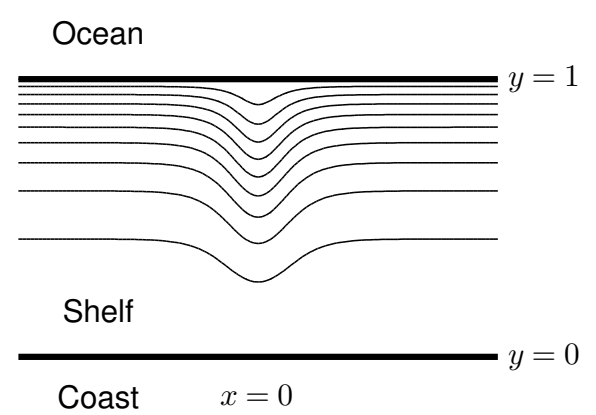

(b)

Figure 1. Isobaths (not drawn to scale) of (a) a submerged ridge $(\alpha<0)$ and (b) a submerged valley $(\alpha>0)$.

We construct this depth profile by introducing a perturbation (to a rectilinear shelf) in the longshore direction

$$
H(x, y)=\exp (2 b p(x, y)), \quad y \in[0,1],
$$

where

$$
p(x, y)=y-1+y(1-y)(\alpha / 2) \epsilon^{2} g(\epsilon x) .
$$

At the coast $(y=0)$, the depth is constant $H_{0}(x, 0)=e^{-2 b}$ and at the shelf-ocean boundary $(y=1)$ is $H_{1}(x, 1)=1$. For this work we choose $g(\epsilon x)=\operatorname{sech}^{2}(\epsilon x)$, so that the maximum perturbation to the Buchwald and Adams (1968) exponential profile occurs in the neighbourhood of $x=0$ and then disappears as $|x| \rightarrow \infty$ (see figures 1 and 2.2). We assume the slowness parameter $\epsilon \ll 1$ and introduce a parameter $\alpha$, which is the amplitude of this depth perturbation. The sign of $\alpha$ determines whether we have a submerged ridge, for $\alpha<0$ (figures 1(a) and 2.2), or a submerged valley, for $\alpha>0$ (figures $1(\mathrm{~b})$ and 2.2). When $a=0$, the bathymetry is rectilinear. Solutions of (2.1) of the form $\Psi(x, y, t)=\operatorname{Re}\{\exp (-\mathrm{i} 2 \Omega \omega t) \psi(x, y)\}$, satisfy

$$
\psi_{x x}+\psi_{y y}-2 b\left(p_{x} \psi_{x}+p_{y} \psi_{y}\right)-2 b \mathrm{i} / \omega\left(p_{y} \psi_{x}-p_{x} \psi_{y}\right)=0
$$

Note from Kaoullas and Johnson (2010a) that for a rectilinear exponential shelf with a Neumann boundary condition at the shelf-ocean boundary, all evanescent modes lie along the curve $k=b / \omega$ (where $k$ is the $x$-wavenumber) and the cutoff frequency of all propagating modes satisfies this relation. If a mode is trapped (in the perturbed channel) it must match in the farfield, to the decaying modes of the unperturbed channel implying that this relationship still holds, fixing $k$ in terms of $\omega$. This important simplification (also used in Postnova and Craster (2008) and Johnson et al. (2011)) reduces the problem to a single eigenvalue $\omega$. If the open ocean boundary condition (2.3) is considered, the relation between $k$ and $\omega$ becomes a trancedental equation resulting in a much harder asymptotic analysis. We look for solutions of the form $\psi(x, y)=\exp (\mathrm{i} b x / \omega) \Phi(x, y)$, and introduce a slow longitudinal variable $\xi=\epsilon x$

$$
\begin{aligned}
\epsilon^{2} \Phi_{\xi \xi}+\Phi_{y y}+\left[2 \epsilon \mathrm{i}(b / \omega)\left(1-p_{y}\right)-2 b \epsilon^{2} p_{\xi}\right] \Phi_{\xi}+\left[\epsilon 2 \mathrm{i}(b / \omega) p_{\xi}-2 b p_{y}\right] \Phi_{y} \\
+\left[(b / \omega)^{2}\left(2 p_{y}-1\right)-\epsilon 2 \mathrm{i}\left(b^{2} / \omega\right) p_{\xi}\right] \Phi=0
\end{aligned}
$$

where

$$
p_{y}=1+(1-2 y)(\alpha / 2) \epsilon^{2} g(\xi), \quad p_{\xi}=y(1-y)(\alpha / 2) \epsilon^{2} g_{\xi}(\xi)
$$


For boundary conditions we take (2.2) and (2.4) giving $\Phi(x, 0)=0$ and $\Phi_{y}(x, 1)=0$ respectively. Following the approach for near cut-off modes of Gridin et al. (2005) and Postnova and Craster (2008) we introduce the following regular asymptotic solutions to $(2.8)$

$$
\begin{aligned}
\Phi(\xi, y) & =\Phi^{(0)}(\xi, y)+\epsilon \Phi^{(1)}(\xi, y)+\epsilon^{2} \Phi^{(2)}(\xi, y)+\cdots, \\
(b / \omega)^{2} & =\mu_{0}+\epsilon \mu_{1}+\epsilon^{2} \mu_{2}+\cdots .
\end{aligned}
$$

These give to leading order

$$
\Phi_{y y}^{(0)}-2 b \Phi_{y}^{(0)}+\mu_{0} \Phi^{(0)}=0
$$

subject to the boundary conditions $\Phi^{(0)}(\xi, 0)=\Phi_{y}^{(0)}(\xi, 1)=0$. The solution for $(2.12)$ is

$$
\Phi^{(0)}=f^{(0)}(\xi) e^{b y} \sin \gamma y, \quad \gamma=\sqrt{\mu_{0}-b^{2}},
$$

and $\mu_{0}$ which gives the cutoff frequency of the rectilinear channel, is determined by

$$
\tan \gamma=-\gamma / b
$$

The function $f^{(0)}(\xi)$ contains the longshore behaviour of the solution and remains to be determined. The equation of order $\epsilon$ is given by

$$
\Phi_{\eta \eta}^{(1)}-2 b \Phi_{\eta}^{(1)}+\mu_{0} \Phi^{(1)}=-\mu_{1} \Phi^{(0)},
$$

subject to the boundary conditions $\Phi^{(1)}(\xi, 0)=\Phi_{y}^{(1)}(\xi, 1)=0$. Multiplying both sides of the equation by $u e^{-2 b y}$, where $u$ is the adjoint of the solution of the homogeneous problem, and integrating from $y=0$ to $y=1$, forces the left-hand side of the equation to vanish and gives the solvability condition (Nayfeh 1993; Postnova and Craster 2008; Johnson et al. 2011)

$$
\mu_{1} \int_{0}^{1} e^{-2 b y} u \Phi^{(0)} \mathrm{d} y=\mu_{1} \int_{0}^{1} \sin ^{2} \gamma y \mathrm{~d} y=0 .
$$

Evaluating the integral gives $\mu_{1}(2 \gamma-\sin 2 \gamma)=0$, forcing $\mu_{1}=0$ (as in Postnova and Craster (2008)), since the term in the brackets is strictly positive, by the definition of $\gamma(2.14)$, which requires $\gamma>\pi / 4$. The solution for (2.15) thus becomes $\Phi^{(1)}=f^{(1)}(\xi) e^{b y} \sin \gamma y$. The order $\epsilon^{2}$ equation is

$$
\Phi_{y y}^{(2)}-2 b \Phi_{y}^{(2)}+\mu_{0} \Phi^{(2)}=-\Phi_{\xi \xi}^{(0)}+\alpha g(\xi)(1-2 y)\left(b \Phi_{y}^{(0)}-\mu_{0} \Phi^{(0)}\right)-\mu_{2} \Phi^{(0)},
$$

subject to $\Phi^{(2)}(\xi, 0)=\Phi^{(2)}(\xi, 1)=0$. The appropriate solvability condition gives the second order ordinary differential equation for $f^{(0)}$,

$$
f_{\xi \xi}^{(0)}+\left[\mu_{2}-V(\xi)\right] f^{(0)}=0,
$$

where

$$
V(\xi)=2 \alpha g(\xi)(b+1)\left(b^{2}+\gamma^{2}\right) \sin ^{2} \gamma / \gamma(2 \gamma-\sin 2 \gamma) .
$$

Equation (2.18) is a one-dimensional Schrödinger equation with $V$ the associated potential and $\mu_{2}$ the eigenvalue. Since the coefficient of $\alpha$ in (2.19) is positive by the definition of $\gamma$, the sign of the potential depends solely on the sign of $\alpha$. Gridin et al. (2005) derive an equation similar to (2.18) for elastic trapped modes and discuss the theoretical consequences. Applying their discussion of the positive operator theory, here, for trapped modes we require that $(2.18)$ has exponentially decaying solutions at infinity, i.e. $f^{(0)} \rightarrow 0$ 
as $|\xi| \rightarrow \infty$. Since trapped modes must have a higher frequency $\omega$ than the cutoff frequency of the unperturbed channel, $\omega_{0}$, we deduce from (2.11) that $\mu_{2}$ must be negative. Multiplying both sides of (2.18) by $f^{(0)}$ and integrating by parts on $(-\infty, \infty)$ gives

$$
\int_{-\infty}^{\infty}\left(f_{\xi}^{(0)}\right)^{2} \mathrm{~d} \xi+\int_{-\infty}^{\infty} V(\xi)\left(f^{(0)}\right)^{2} \mathrm{~d} \xi=\mu_{2} \int_{-\infty}^{\infty}\left(f^{(0)}\right)^{2} \mathrm{~d} \xi .
$$

If $V$ is positive, the left-hand side of (2.20) is positive forcing $\mu_{2}$ to be positive, which contradicts the fact that trapped modes must have a higher frequency than the cutoff frequency. Also by inspecting equation (2.18), the second term vanishes as $|\xi| \rightarrow \infty$ and if $\mu_{2}$ is non-negative it leaves an ODE with propagating solutions, implying that trapped modes cannot exist. If $V$ is negative, implying that $\alpha<0$, a negative eigenvalue might exist (depending on the magnitude of $\alpha$ ). Hence, in this problem there is a possibility of existence of trapped modes, in the case of a submerged ridge $(\alpha<0)$, while submerged valleys (in this problem) cannot support trapped modes.

Taking the shelf-ocean boundary to be impermeable i.e. a Dirichlet boundary condition, the flow is that along a straight, infinite channel with increasing cross-channel depth and a depth perturbation at the middle of the channel. The above manipulations give $\gamma=n \pi, \quad n=1,2, \ldots$ and $V(\xi)=\alpha b g(\xi)$. Since the terms multiplied with $\alpha$ are always positive, the same conclusions for trapping to occur still hold.

\subsection{Analytical solution}

Equation (2.18) is to be solved numerically in general, however, for the specific choice of the function $g(\xi)=\operatorname{sech}^{2}(\xi)$, it can be solved analytically following Landau and Lifshitz (1991) as

$$
f^{(0)}(\xi)=\cosh ^{m-s}(\xi) F(-m, 2 s+1-m ; s+1-m ;(1-\tanh \xi) / 2),
$$

where

$$
s=(\sqrt{1-4 \alpha \beta}-1) / 2, \quad \beta=(b+1)\left(b^{2}+\gamma^{2}\right) \sin ^{2} \gamma / \gamma(2 \gamma-\sin 2 \gamma),
$$

and

$$
\mu_{2}=-(s-m)^{2}, \quad 0 \leq m<s,
$$

and $F$ is the hypergeometric function. Since $-m$ is a negative integer, $F$ is a polynomial of degree $m$. The condition that $s>m$ gives the following lower bound on the parameter $\alpha$

$$
-\alpha>\left[(2 m-1)^{2}-1\right] / 4 \beta .
$$

Higher modes (in the along-stream direction) need a larger value of $|\alpha|$, in order to become trapped, and corresponds to the fact that $\alpha$ is the magnitude of the potential $V$. This implies that a larger amplitude in the depth perturbation is needed. Higher modes in the cross-stream direction, affect relation (2.24) only through $\beta$. It is remarkable that for the first transverse mode, when $m=1$, condition (2.24) becomes that $\alpha>0$, which is independent of $\beta$. Hence, as long as there is a perturbation in depth, the first transverse mode becomes trapped and an infinite number of cross-stream modes can exist as well. As the number of cross-stream modes goes to infinity, from (2.14)

$$
\gamma \rightarrow \pi(2 q-1) / 2, \quad q \in \mathbb{N}, \quad q \gg 1,
$$

and $\beta \rightarrow 1 / 2(b+1)$, implying that for each $m$ there is an infinite number of cross-stream trapped modes. Similar conclusions, regarding higher modes, can be drawn for the curved oceanic waveguide considered by Postnova and Craster (2008) and Johnson et al. (2011). 


\subsection{Numerical method for the full problem}

In this section we describe a highly accurate pseudospectral method which is used to solve the full partial differential equation (2.7) and to calculate the eigenfrequency $\omega$ for each trapped mode (see Boyd 2000; Trefethen 2000). This method is independent of the small depth changes (along-shore) limitation and thus will be used to provide verification of the accuracy of the asymptotic scheme and to investigate a wider range of parameters. It involves expanding the unknown solution of the equation as a global interpolant, and thus deriving the differentiation matrix corresponding to each interpolant (Weideman and Reddy 2000). A similar method is applied to elastic modes in Gridin et al. (2005).

Looking for solutions of $(2.7)$ of the form $\psi(x, y)=\exp (\mathrm{i} b x / \omega) \Phi(x, y)$ and considering the profile (2.6) gives

$$
\begin{aligned}
\omega^{2}\left[\Phi_{x x}+\Phi_{y y}-2 b\left(p_{x} \Phi_{x}+p_{y} \Phi_{y}\right)\right]+2 b \mathrm{i} \omega\left[\left(1-p_{y}\right) \Phi_{x}+p_{x}\right. & \left.\left(\Phi_{y}-b \Phi\right)\right] \\
& +b^{2}\left(2 p_{y}-1\right) \Phi=0 .
\end{aligned}
$$

We will be solving the equation above numerically for the eigenvalue $\omega$, subject to Dirichlet condition at the coast, Neumann condition at the shelf-ocean boundary, and vanishing disturbance in the farfield. From the definition of $g(\epsilon x)$ we get that $H(x, y)=H(-x, y)$, which in turn implies that if $\psi(x, y)$ is a solution to $(2.7)$, so is $\psi^{*}(-x, y)$ and $\psi(x, y)=$ $\psi^{*}(-x, y)$, where $*$ denotes the complex conjugate. Due to this symmetry it is sufficient to solve for $x \geq 0$. The geometry of the straight coastline problem is reduced to a semi-infinite strip of fixed width, thus in the cross-shore direction $y \in[0,1]$ we will use a Chebyshev method. In the along-shore direction $x \in[0, \infty]$ we will again use a Chebyshev method on the truncated domain, say $x \in[0, L]$. L must be large enough to allow for the solution to decay but to maintain resolution as $L$ is increased the number of alongshore points needs to increase. If a trapped mode exists, it matches in the farfield $(x \rightarrow \infty)$ to a decaying mode of the rectilinear shelf. Then, the decay rate of the trapped mode is proportional to $\exp (-\operatorname{Im} k)$ where $k$ is the $x$-wavenumber of the rectilinear shelf, which can be obtained analytically (Buchwald and Adams 1968).

The frequency $\omega$, of trapping is thus calculated by solving equation (2.26) numerically on a spectral grid. This gives an eigenvalue problem solved using a shifted method, given in Appendix A.

\subsection{Comparison of numerical and asymptotic results}

Let $\omega_{m, n}$ be the frequency of the $(m, n)$ trapped mode where $m$ is the mode number in the $x$-direction and $n$ in the $y$-direction. To get a direct comparison between the asymptotic and numerical methods we need to define the numerical value of $\mu_{2}$ as $\mu_{2_{N}}=$ $\left[\mu_{0}-\left(\omega_{0}+\lambda\right)^{-2}\right] / \epsilon^{2}$.

The numerical method is very accurate indeed, with $\mu_{2 N}$ for the eigenvalue $\omega_{1,1}$ achieving an accuracy of five significant figures for $M=20, N=10$, which implies an accuracy of eight digits (for $\epsilon=0.1$ ) for $\omega$. Higher modes require much higher resolution for similar accuracy with $\omega_{2,3}$ requiring $M=66, N=22$.

Direct comparison between the two methods is demonstrated in table 1. The asymptotic eigenvalues are indeed very accurate, with the relative error in $\mu_{2}$ well below $10 \%$. This is due to the fact that in the asymptotic expansions we have discarded the $O\left(\epsilon^{3}\right)$ terms implying that $\mu_{2_{A}}$ should be accurate at least to $O(\epsilon)$, which is precisely what emerges from the numerics in Table 1 . The relative error is increasing for higher modes, since the coefficients of the $O\left(\epsilon^{3}\right)$ terms could become more important for higher modes.

In figure $2, \Phi$ is plotted as a function of the variable $x$ at $y=1$, for the modes $\omega_{1,1}$ and $\omega_{2,1}$, demonstrating good agreement between the asymptotic and the numerical method. 


$\begin{array}{cccccccc}\mathrm{n} & \mathrm{m} & \omega & b / \omega_{0} & b / \omega & \mu_{2} & \mu_{2} & \text { r.error } \\ 1 & 1 & 0.6588161295 & 3.039605122 & 3.03574838 & -2.3431 & -2.3366 & -0.0028 \\ 1 & 2 & 0.6580805227 & - & 3.03914176 & -0.2816 & -0.2807 & -0.0032 \\ 2 & 1 & 0.3660655837 & 5.466023907 & 5.46350187 & -2.7564 & -2.7137 & -0.0158 \\ 2 & 2 & 0.3659233772 & - & 5.46562511 & -0.4359 & -0.4250 & -0.0259 \\ 3 & 1 & 0.2398712074 & 8.339536263 & 8.33780770 & -2.8827 & -2.7844 & -0.0352 \\ 3 & 2 & 0.2398298861 & - & 8.33924426 & -0.4870 & -0.4609 & -0.0562\end{array}$

TABLE 1. The trapped frequencies $\omega$ as calculated from the asymptotic method and a comparison of $\mu_{2}$ from the asymptotic method, $\mu_{2_{A}}$, and the numerical method, $\mu_{2_{N}}$. Here $\alpha=-\pi / 2$ and $\epsilon=0.1$.

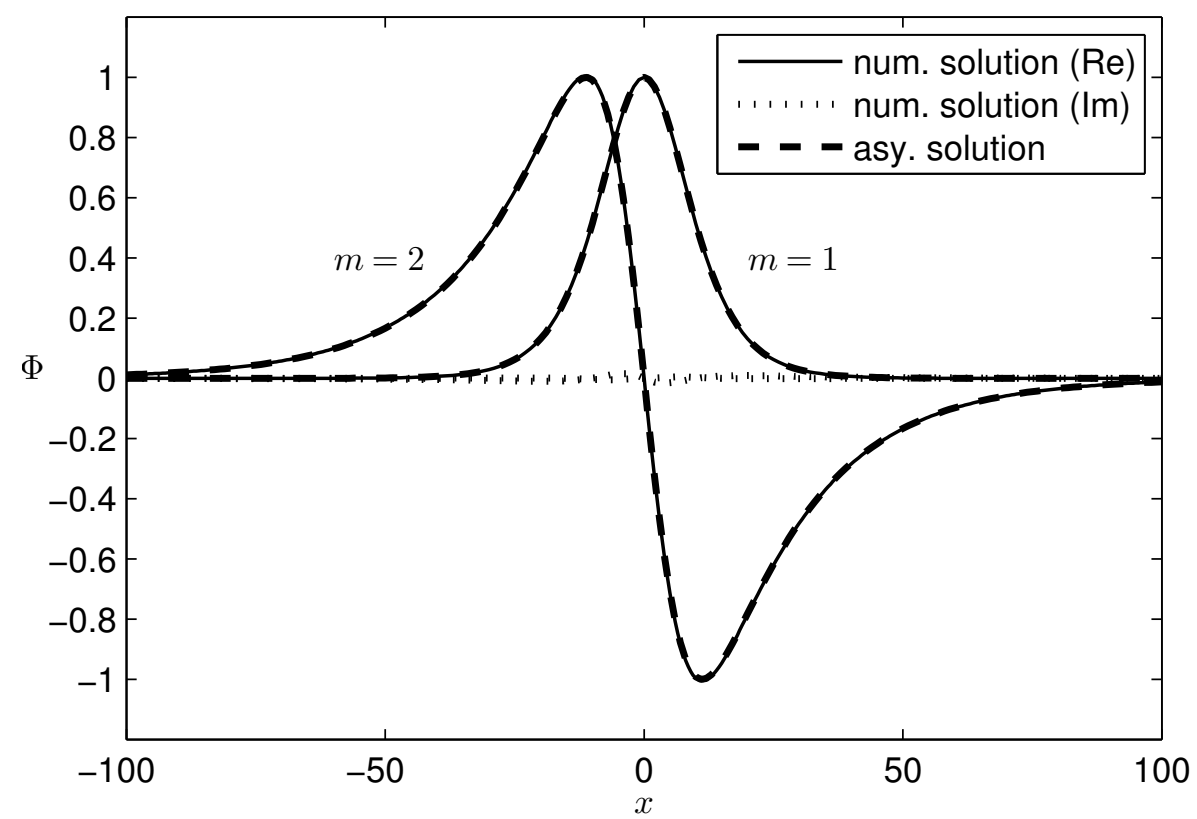

FiguRE 2. $\Phi(x, 1)$ generated by the spectral method (solid line) and the asymptotic method (dashed line) for the $\omega_{1,1}$ and $\omega_{2,1}$ modes. Here $b=2, \alpha=-\pi / 2$, and $\epsilon=0.1$.

The imaginary part of $\Phi$, with a magnitude of $O\left(\epsilon^{3}\right)$, is also shown for the numerical solution, implying that a small proportion of the phase is still present in the full problem. It is non-zero because the phase changes slightly as the depth changes in $x$ over a distance of $\epsilon^{-1}$ and vanishes as $\epsilon$ goes to zero. The imaginary part of the asymptotic solution is identically zero, for the present order of accuracy. Figure 3(a) is a contour plot of the envelope of the trapped mode $\omega_{1,1}$ and figure $3(\mathrm{~b})$ for the $\omega_{2,3}$ mode. The disturbance is concentrated above the perturbed section of the shelf and decays exponentially along the shelf as expected.

We have demonstrated the existence of trapping for a submerged ridge, where the shelf had finite extent (limited to [0,1]). As shown in Johnson and Kaoullas (2011) both the wall or the slope effect alone, or combined could show trapping. To give a better understanding of the underlying physics which lead to trapping we will consider the 


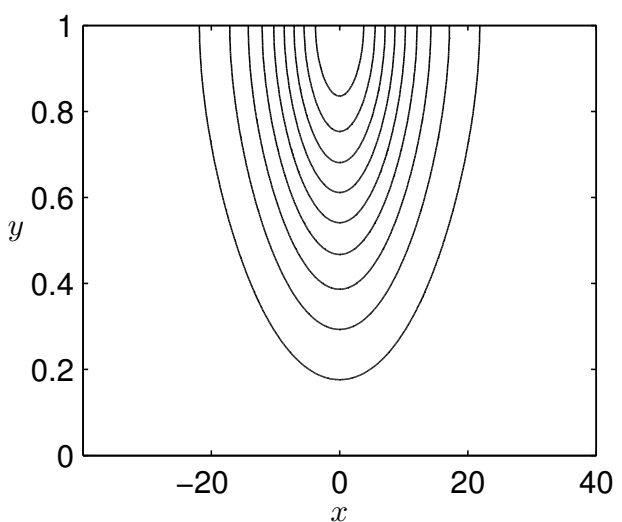

(a)

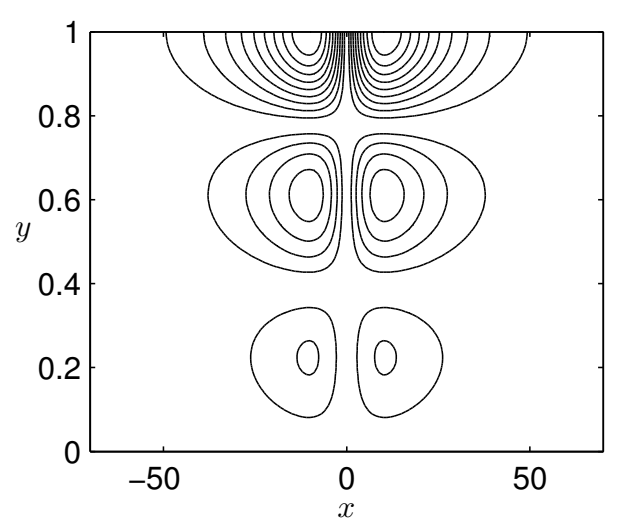

(b)

Figure 3. $|\Phi|$ for (a) $(1,1)$ mode and (b) $(2,3)$ mode for parameters $b=2, \alpha=-\pi / 2$, and $\epsilon=0.1$.

following (more general) rectilinear depth profile in $y>0$ (Johnson and Kaoullas 2011)

$$
H(y)=H_{0}+\left(1-H_{0}\right)\{1+\tanh [(y-c) / s]\} / 2 .
$$

$H_{0}$ is the depth at the coastal wall, the depth in the open ocean is 1 and $c$ gives the distance of shelf-break (maximum slope) to the wall and $s$ measures the slope of the shelf-break. Figure 4(a) shows the profiles for shelf-breaks at $c=4,5,6, H_{0}=0.25$ and $s=1$, while figure 4(b) the corresponding sections of the dispersion relations between the frequency $\omega$ of the fundamental cross-shelf mode and its along-shore wavenumber $k$ near cut-off frequencies, computed by the spectral method of Kaoullas and Johnson (2010a). As the distance of the shelf-break form the wall increases, so does the cut-off frequency since increasing the distance between the shelf-break and the coast weakens the boundary constraint (Johnson 1989) giving higher propagating frequencies at any fixed wavenumber. Thus if a shelf has a finite region where the shelf-break is further from the wall than in the farfield i.e. a submerged ridge, the cutoff frequency of waves is raised locally, while in the farfield it remains unchanged, allowing for trapping. On the other hand submerged valleys formed when the shelf-break is locally closer to the wall do not support tCSWs.

Figure 5(a) shows the profile (2.27) for coastal depths $H_{0}=0.25$, a shelf-break at $c=5$ and slope widths $s=0.5,1.0,1.5$, and Figure 5(b) shows sections, near the maximum propagating frequency, of the dispersion diagram for the fundamental cross-shelf mode for these slope widths $s$. It is clear that for wider slopes the frequencies of locally propagating modes are reduced and so locally trapped modes are not possible, while if the the shelf steepens locally and then widens in the farfield, forming a submerged valley it allows for tCSWs. The latter type of geometry is discussed in greater detail in section 3 . For the topography (2.5) considered here, it is a combination of these two factors that gives the existence of trapped modes.

Similar dispersion curves can also be drawn to demonstrate the curvature geometric effect (see section 3) and are given in Kaoullas and Johnson (2010b) where it is noted that by bending locally a straight rectilinear shelf towards the coast increases the cut-off frequency in the curved section. Questions still remain on how trapping will be affected when considering a shelf which extends into an open ocean. 


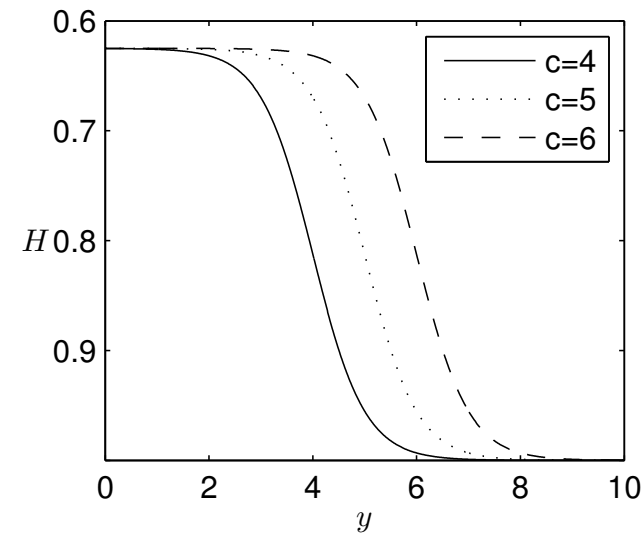

(a)

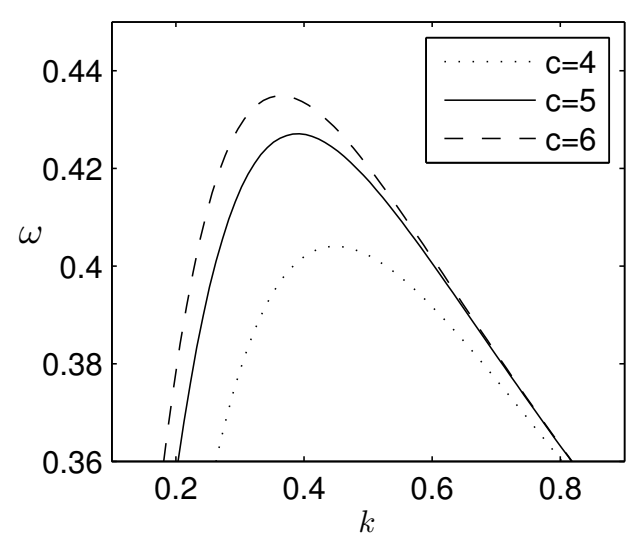

(b)

Figure 4. (a) Depth profiles and (b) dispersion curves of the first propagating mode for shelf breaks at $c=4$ (dotted line), $c=5$ (solid line) and $c=6$ (dashed line) for the profile (2.27). Here, $s=1$ and $H_{0}=0.25$.

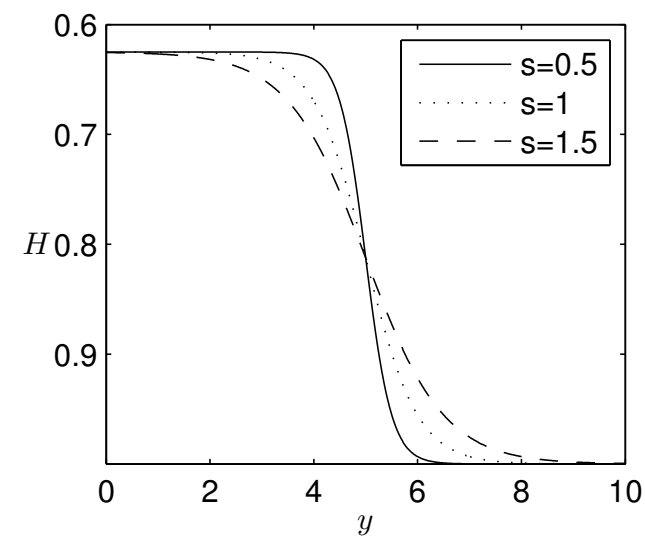

(a)

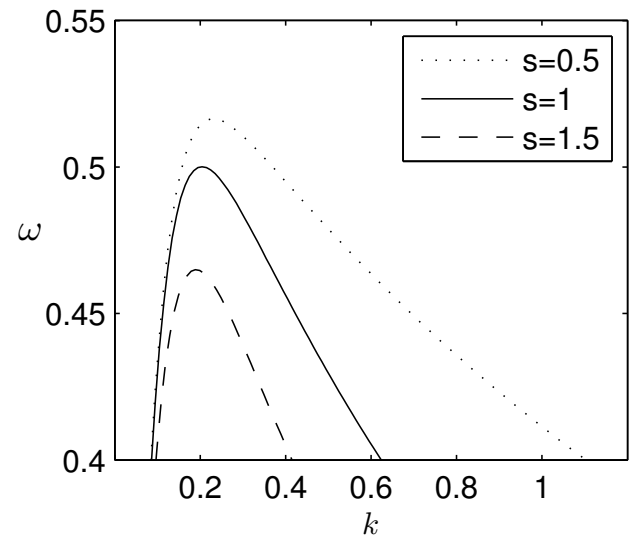

(b)

Figure 5. (a) Depth profiles and (b) dispersion curves of the first propagating mode for shelf widths $s=0.5$ (dotted line), $s=1$ (solid line) and $s=1.5$ (dashed line) for the profile (2.27). Here, $c=5$ and $H_{0}=0.25$.

\subsection{Open Ocean Trapping}

Trapped CSWs in smooth coastlines are established by JLP and then by Postnova and Craster (2008) and Kaoullas and Johnson (2010b). The most serious approximation in these papers is that the open ocean is omitted by applying a Neumann boundary condition at the shelf-ocean boundary, thus reducing the problem to a strip. The same approximation is necessary to obtain the asymptotic results for a shelf with alongshore depth variations in $\S 2.2$. Even though this approximation simplifies these problems, as pointed in Kaoullas and Johnson (2010b) it leads to discontinuous velocity profiles. Adapting the asymptotic methods for the curved coastline and for the straight non-rectilinear shelf is not easy since an extra term involving $k$ appears in the relationship $k=b / \omega$ so fixing $k$ in terms of $\omega$ is more difficult. Also, the mode-matching method in Kaoullas and Johnson (2010b) fails, since the set of cross-shelf eigenfunctions is not complete and obtaining a rigorous result as in JLP is still an open research problem. 


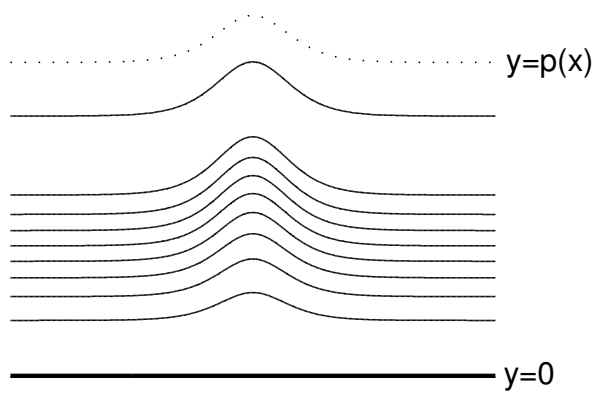

(a)

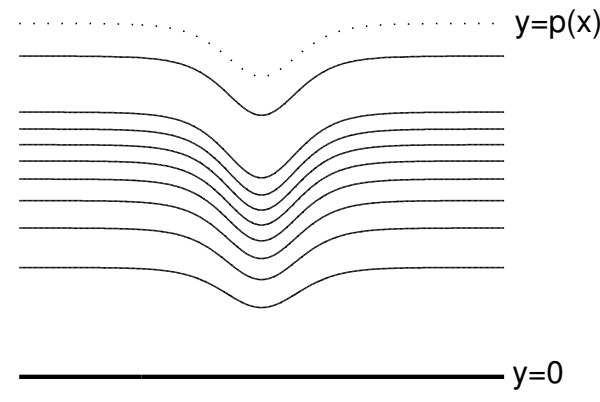

(b)

Figure 6. Isobaths of (a) a submerged ridge $(\alpha>0)$ and (b) a submerged valley $(\alpha<0)$.

Nevertheless, we can still construct numerically trapped modes for these two problems and assess the accuracy of the Neumann shelf-ocean boundary condition using a pseudospectral method similar to that in $§ 2.4$. Consider an infinite, straight coastline as in $\S 2.2$, where the nonrectilinear shelf extends into an open ocean. The governing equation, with the corresponding boundary condition in the infinite ocean can be written as

$$
\begin{array}{r}
H\left(\psi_{x x}+\psi_{y y}\right)-\left(H_{x} \psi_{x}+H_{y} \psi_{y}\right)-\mathrm{i} / \omega\left(H_{y} \psi_{x}-H_{x} \psi_{y}\right)=0, \\
\psi=0, \quad \text { at } y=0, \\
\nabla \psi \rightarrow 0, \quad \text { at } y \rightarrow \infty .
\end{array}
$$

Since the governing equation reduces to Laplace's equation in the open ocean, the last boundary condition is equivalent to $\psi \rightarrow 0$ as $y \rightarrow \infty$. Extending the exponential topography (2.5), into a constant ocean gives a continuous but not differentiable topography function and thus seriously decreases the accuracy of the spectral method (Trefethen 2000). Hence, we construct the (everywhere) smooth topography (shown in figure 6) as

$$
H(x, y)=\left\{\begin{array}{lr}
1-\left(1-H_{0}\right) \exp \left(-\left|y^{2}-p(x)^{2}\right|^{-s}+p(x)^{-2 s}\right), & 0 \leq y \leq p(x), \\
1, & y \geq p(x),
\end{array}\right.
$$

where $p(x)=\left(1+\alpha \operatorname{sech}^{2} \epsilon x\right) /(1+\alpha)$. The depth at the coast is $H_{0}$ and the depth of the ocean is 1 . If $\alpha>0$ (see figure 6(a)), we get the submerged ridge case and if $\alpha<0$ (see figure 6(b)), the submerged valley case, where no trapped modes can be supported. The disadvantage, using this depth profile instead of (2.5) is that the governing equation in far-field does not have constant coefficients, hence the cross-stream modes have no simple structure and it is not straightforward to factorise the phase from the problem, requiring greater computational effort. We discretise the domain, over the half plane, as in Appendix A, resulting in a grid of $N$ points in the $y$-direction and $M$ in the $x$ direction. We use a Hermite method in the $x$-direction, which is more appropriate (i.e. more accurate) than a Chebyshev method on the real line, since the latter clusters points near the end of the interval and in the $y$-direction we use a Laguerre method. Similarly to $§ 2.3$, we calculate the eigenvalue of trapping using a shifted eigenvalue method. The Dirichlet condition at the coast is implemented as in Appendix A.

When dealing with unbounded domains it is often very important to choose a sensible scaling factor $\beta$ (Tang 1993; Weideman and Reddy 2000; Shen and Wang 2009). Let $x_{j}$ be the collocation points of the Hermite method, $u\left(x_{i}\right.$ the solution at these points and let $\delta_{x}$ 


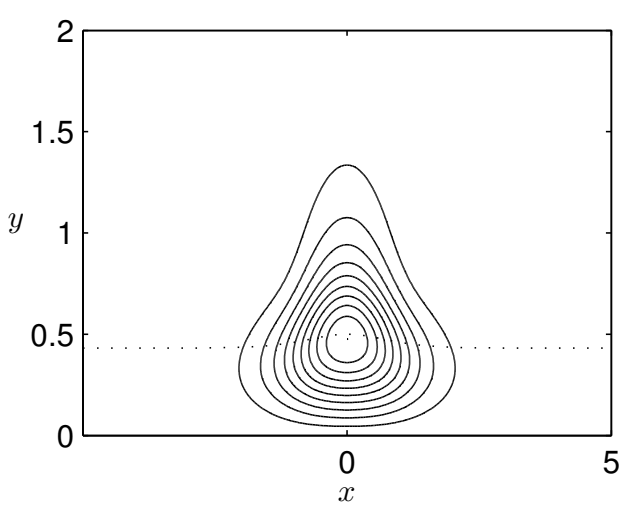

(a)

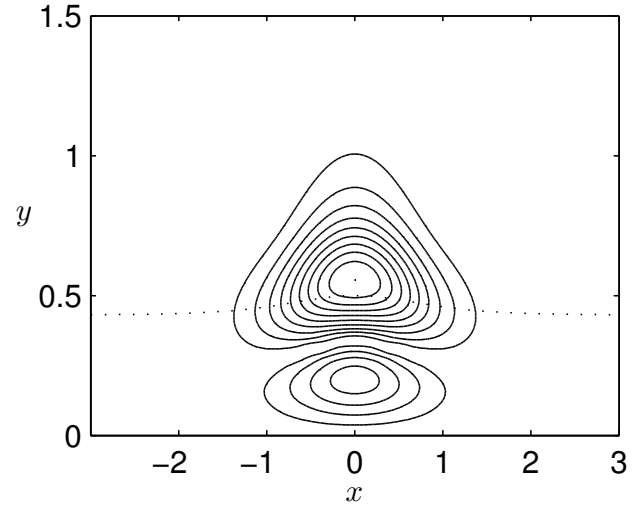

(b)

Figure 7. Contour plot of $|\psi|$ for (a) the mode $\omega_{1,1}$, and (b) the mode $\omega_{1,2}$. The dotted line shows the position of the shelf break. Here, $s=2, \alpha=0.16$ and $\epsilon=1$.

be an accuracy threshold such that $\left|u\left(x_{i}\right)\right|<\delta_{x}$, and $\left|x_{i}\right| \leq L_{x}$, for all $1 \leq i \leq M$. Then, we set the scaling factor $\beta_{H}=x_{M} / L_{x}$, where $x_{M}$ is the largest Hermite collocation point i.e. the $M$ th zero of the Hermite polynomial of degree $M$ (Tang 1993; Abramowitz and Stegun 1964). If a trapped mode exists, it matches in the farfield $(x \rightarrow \infty)$ to a decaying mode of the rectilinear shelf. Then, the decay rate of the trapped mode is $\exp (-\operatorname{Im} k)$ where $k$ is the $x$-wavenumber of the rectilinear shelf, which can be calculated using the numerical method in Kaoullas and Johnson (2010a). Hence, we can determine $L_{x}$ and the corresponding $\delta_{x}$. Similarly for the Laguerre interpolant we define $\beta_{L}=y_{N} / L_{y}$, where $y_{N}$ is the largest Laguerre collocation point i.e. the $N$ th zero of the Hermite polynomial of degree $N$ (Shen and Wang 2009; Szegö 1975). The decay rate of a trapped mode in the open ocean is $\exp (-\operatorname{Re} k)$, and thus $L_{y}$ and $\delta_{y}$ can be determined.

\subsection{Numerical results}

As before, we let $w_{m, n}$ be the frequency of the $(m, n)$ trapped mode, $m$ is the mode number in the longshore direction and $n$ in the cross-shore direction. For the fundamental eigenfrequency $\omega_{1,1}$, with a cutoff frequency $\omega_{0}=0.40793063$, an accuracy of five digits is achieved for $M=44, N=34$, for near optimal values of $\beta$ and the decay rate of the corresponding eigenfunction $\psi$ is approximately $\exp (-1.75)$ in the longshore direction and approximately $\exp (-5.66)$ in the cross-shore direction. Figures $7(\mathrm{a})$ and $7(\mathrm{~b})$ are contour plots of $|\psi|$ for the trapped modes $\omega_{1,1}$, and $\omega_{1,2}$, respectively. Most of the wave disturbance is concentrated along the submerged ridge, with the maximum disturbance being near the shelf break.

The boundary condition in the open ocean, that $\nabla \psi \rightarrow 0$ as $y \rightarrow \infty$ is equivalent to $\psi \rightarrow 0$ as $y \rightarrow \infty$, which implies that both the Dirichlet and Neumann condition are equivalent as $y \rightarrow \infty$. It can be shown using the variational principle as in JLP that for a straight, rectilinear, oceanic waveguide the top of the essential spectrum i.e. the cutoff frequency is always higher for a Neumann boundary condition (at the shelf-ocean boundary) compared to a Dirichlet boundary condition. Also, from Johnson (1989) the Dirichlet condition gives a lower bound on the frequency of trapping $\omega_{D}$ and the Neumann condition an upper bound $\omega_{N}$ :

$$
\omega_{D} \leq \omega \leq \omega_{N}
$$

If we impose a Dirichlet (Neumann) condition at $L_{y}=1$, i.e. at the shelf-ocean boundary 


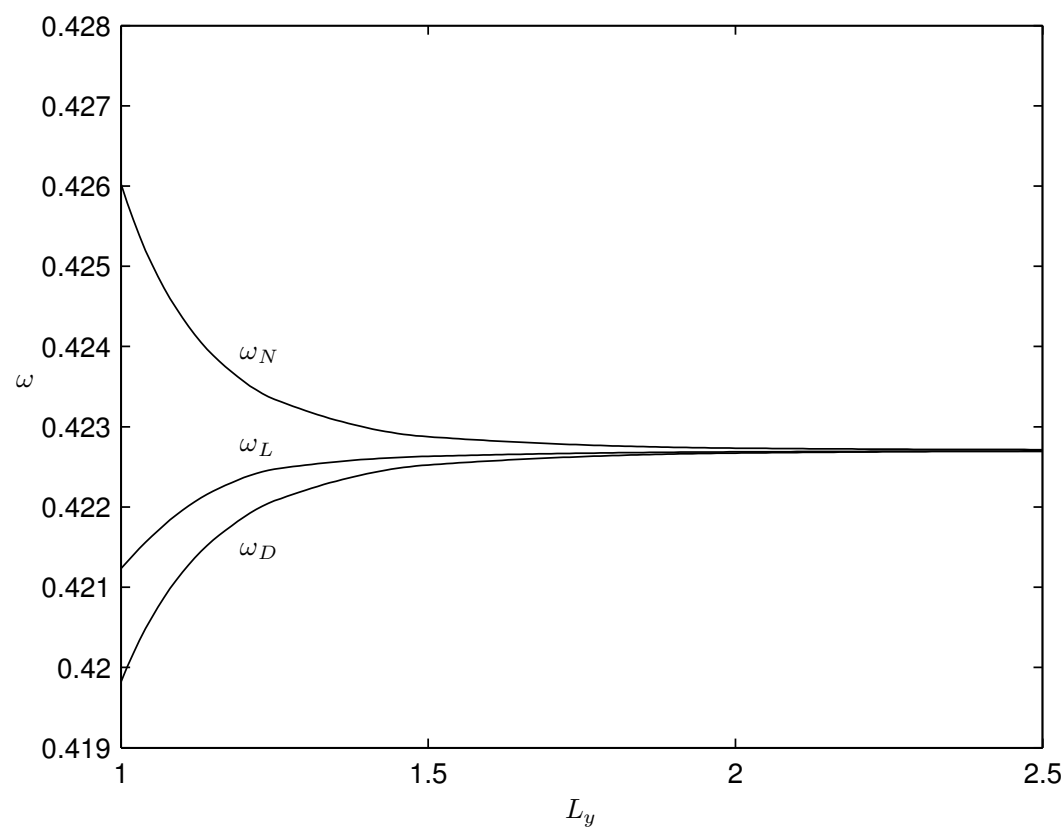

Figure 8. Convergence of the mode $\omega_{1,1}$, for the straight coast, for the Chebyshev grid with Dirichlet b.c. $\left(\omega_{D}\right)$ and Neumann b.c. $\left(\omega_{N}\right)$ and the Laguerre grid $\left(\omega_{L}\right)$ for increasing $L_{y}$. Here, $s=2, \alpha=0.16$ and $\epsilon=1$.

and then increase $L_{y}$ gradually to infinity, the frequency of trapping $\omega_{D}\left(\omega_{N}\right)$ should converge to the same value $\omega$. To implement this numerically we employ a Chebyshev method.

We demonstrate these bounds for the fundamental trapped mode in figure 8. It is evident that the frequencies for the two boundary conditions are converging as $L_{y} \rightarrow \infty$. Additionally, the frequency obtained with the Laguerre grid $\omega_{L}$ shows that the accuracy of the Laguerre grid is slightly better than the accuracy of the Chebyshev grid. Note however, that to obtain an accuracy of 5 digits when $L_{y}=2.5$ requires 40 points for the Dirichlet and Neumann boundary conditions but only 34 points for the Laguerre scheme showing that the Laguerre method should be the method of choice for these problems.

\section{Salient shelf geometry characteristics}

Johnson and Kaoullas (2011) note three characteristics of the shelf geometry, namely shelf curvature, the wall effect and the slope effect which alone or combined are responsible for trapping. The first characteristic is examined alone in the case of a strip (JLP; Postnova and Craster (2008); Kaoullas and Johnson (2010b)) and the other two are examined combined (in section 2) for a strip and a semi-infinite domain. In this section we demonstrate that each characteristic alone is sufficient for the existence of trapped modes in semi-infinite domains.

\subsection{Isobath curvature - Curved Coastline}

As shown in JLP and Postnova and Craster (2008) and in Kaoullas and Johnson (2010b), a trapped mode in a curved oceanic waveguide (with rectilinear topography) exists when 


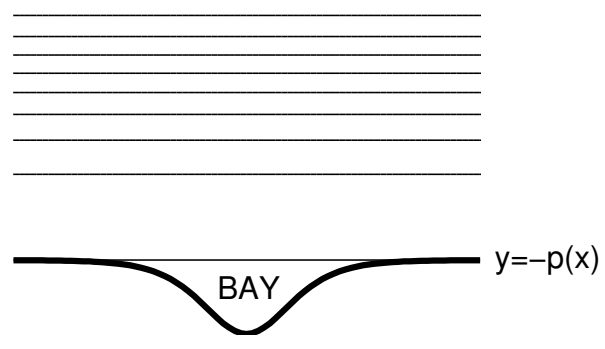

(a)

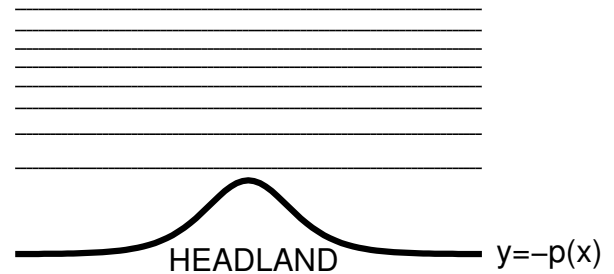

(b)

Figure 9. Isobaths of (a) a bay $(\alpha>0)$ and (b) a headland $(\alpha<0)$. The thick solid line denotes the coastline and the dotted line the shelf-break.

the guide is bent towards the coast. An analogous result should be obtained when the shelf extends into an open ocean.

Consider a curved, rectilinear shelf, which extends into an open ocean. Introducing the curvilinear coordinate system $(\xi, \eta),-\infty \leq \xi \leq \infty, \eta \geq 0$ similarly to JLP, we can write the governing equation (2.28), with the corresponding boundary conditions as

$$
\begin{array}{r}
H\left(p^{2} \psi_{\xi \xi}+\psi_{\eta \eta}-p^{3} \eta \delta_{\xi \xi} \psi_{\xi}+p \delta_{\sigma} \psi_{\eta}\right)-H_{\eta} \psi_{\eta}-i p / \omega H_{\eta} \psi_{\xi}=0, \\
\psi=0, \text { at } \eta=0, \\
\nabla \psi \rightarrow 0, \quad \text { at } \eta \rightarrow \infty
\end{array}
$$

where

$$
p=\left(1+\eta \delta_{\xi}\right)^{-1},
$$

and $\delta_{\xi}=\alpha \operatorname{sech}^{2}(\epsilon x)$ is the curvature (following the definition in JLP). We shall investigate the existence for trapped modes numerically for the following topography

$$
H(\eta)=\left\{\begin{array}{lr}
1-\left(1-H_{0}\right) \exp \left(-\left|\eta^{2}-1\right|^{-s}+1\right), & 0 \leq \eta \leq 1, \\
1, & \eta \geq 1,
\end{array}\right.
$$

where the depth at the coast is $H_{0}$ and the depth at the open ocean is 1 . Even though this topography is rectilinear in the $(\xi, \eta)$ plane, $(3.1)$ differs from $(2.28)$ and thus gives the possibility of trapping.

\subsection{Wall effect - Bay Trapping}

As noted in Johnson and Kaoullas (2011) increasing (decreasing) the distance of the shelfbreak from the coast increases (decreases) the cutoff frequency of propagating modes. A straight rectilinear shelf, extending into a flat open-ocean, is considered (which does not support trapped modes) and a flat bay or a headland (of finite extent) is added to the coast, in the vicinity of $x=0$ as in figure (9). Adding a bay raises locally the maximum frequency that waves can propagate for any fixed along-shore wavenumber, while the cutoff frequency is unchanged hence allowing modes to be trapped in the bay region. Adding a headland lowers locally this maximum frequency and no trapping appears. Consider the topography 


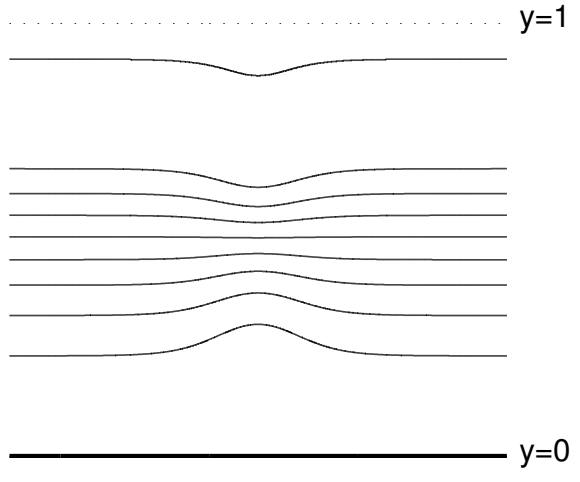

(a)

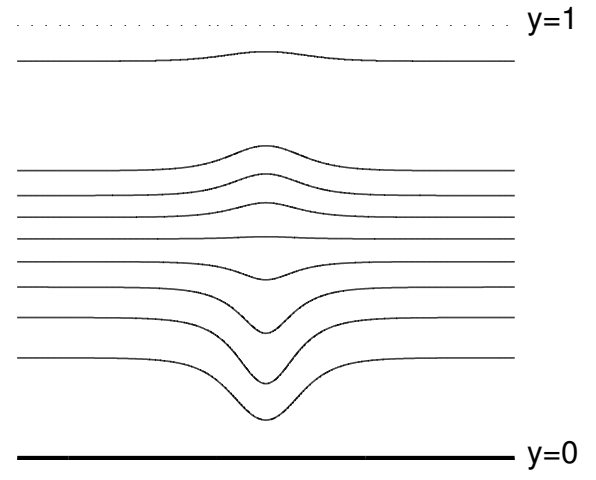

(b)

Figure 10. Isobaths of (a) a valley $(\alpha>0)$ and (b) a ridge $(\alpha<0)$.

$$
H(x, y)=\left\{\begin{array}{lr}
H_{0}, & y \leq-1 \\
1-\left(1-H_{0}\right) \exp \left(1-|y(y+2)|^{-s}\right), & -1 \leq y \leq 0 \\
1, & y>0
\end{array}\right.
$$

where $p(x)=1+\alpha \operatorname{sech}^{2} x$ and $H_{0}$ is the depth at the coast and the depth of the ocean is 1 . The sign of $\alpha$ distinguishes between bays $(\alpha>0$, see figure $9(\mathrm{a}))$ and headlands $(\alpha<0$, see figure $9(\mathrm{~b}))$. We introduce the new co-ordinate $\eta$ as

$$
\eta=y / p(x)
$$

The governing equation (2.28) then becomes

$$
\begin{gathered}
H \triangle \psi-\left(H_{x}-\eta\left(p_{x} / p\right) H_{\eta}\right) \psi_{x}-\left(p^{-2}\left(1+\eta^{2} p_{x}^{2}\right) H_{\eta}-\eta\left(p_{x} / p\right) H_{x}\right) \psi_{\eta}- \\
\mathrm{i} / \omega p\left(H_{\eta} \psi_{x}-H_{x} \psi_{\eta}\right)=0,
\end{gathered}
$$

subject to $\psi=0$ at $y=-p(x)$ and $\psi \rightarrow 0 y \rightarrow \infty$.

\subsection{Slope Effect - Valley Trapping}

Similarly to the wall-effect, increasing (decreasing) the slope of the shelf increases (decreases) the cutoff frequency of locally propagating modes for a fixed wavenumber. Consider the profile shown in figure 10

$$
H(x, y)=\left\{\begin{array}{lr}
1-\left(1-H_{0}\right) \exp \left(p(x, y)^{-2 s}-\left|y^{2}-p(x, y)^{2}\right|^{-s}\right), & 0 \leq y \leq 1 \\
1, & y \geq 1
\end{array}\right.
$$

where

$$
p(x, y)=1+\alpha(y-1)(y-0.5) \operatorname{sech}^{2} x,
$$

and the governing equation is (2.28) - (2.30). With this profile the slope of the shelf is changed locally while keeping the distance of the shelf-break from the coast constant everywhere. Here, trapping is only expected for $\alpha>0$. 


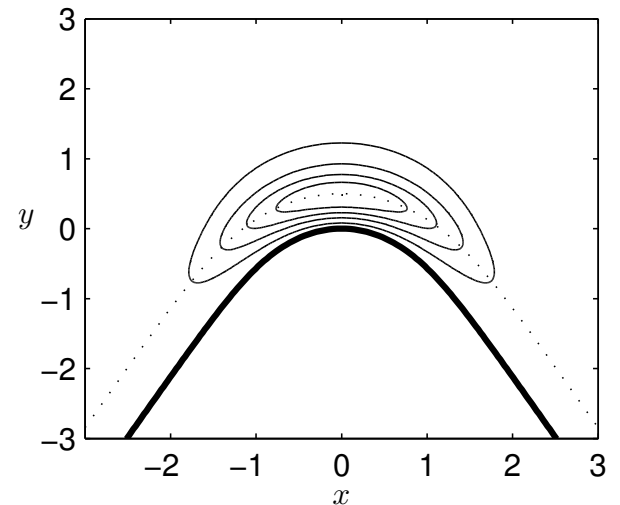

(a)

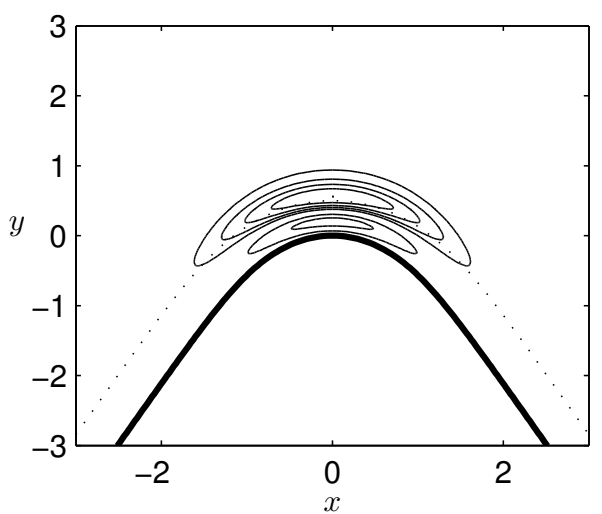

(b)

Figure 11. Contour plot of $|\psi|$ for (a) the mode $\omega_{1,1}$ and (b) the mode $\omega_{1,2}$. The dotted line shows the position of the shelf break and the solid line denotes the coast.

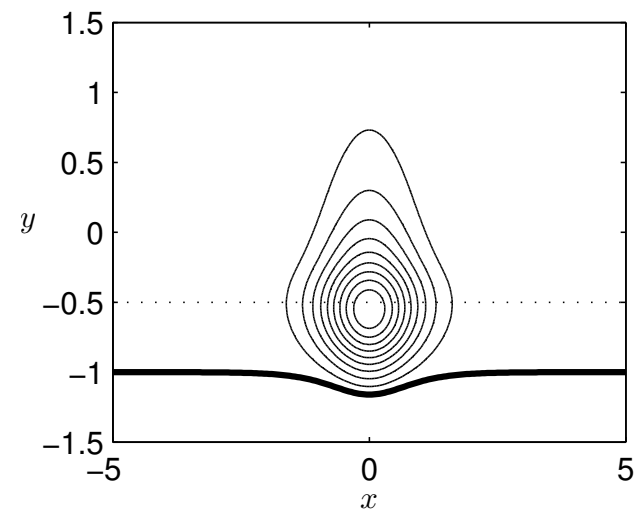

(a)

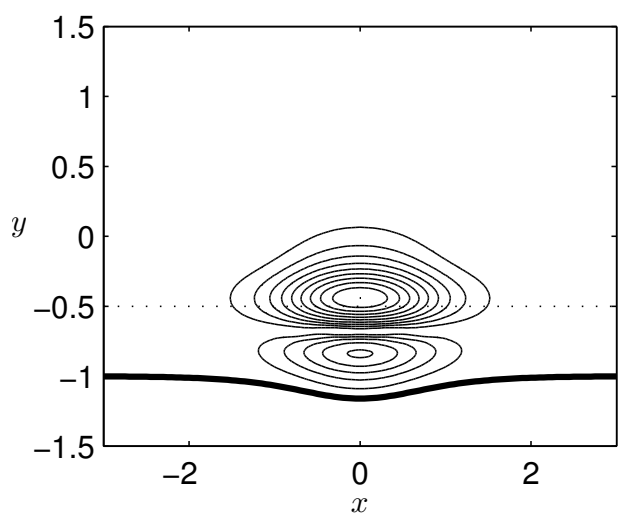

(b)

FIgURE 12. Contour plot of $|\psi|$ for (a) the mode $\omega_{1,1}$ and (b) the mode $\omega_{1,2}$ for the bay problem. The thick solid line denotes the coastline and the dotted line the shelf-break.

\subsection{Results}

Finding numerical solutions of equations (3.1), (3.8), and (2.28) requires only a slight modification to the numerical method presented in section 2, so details will be omitted. A similar convergence to the straight problem in section 2 is achieved for the curved, bay and slope problems for the fundamental eigenfrequency $\omega_{1,1}$. Again the Dirichlet and Neumann approximations, coincide with the unapproximated boundary condition,with increasing the cross-shelf domain size and still capture the main dynamics of trapping and give good qualitative results. Figures 11, 12, and 13 show contour plots of $|\psi|$ of the trapped modes $\omega_{1,1}$ and $\omega_{1,2}$, for the curved problem, bay and slope problem respectively.

The disturbance is concentrated along the shelf-break i.e. over the region of maximum slope and decays exponentially both across the shelf away from the shelf-break and along the coast away from the perturbed region. This accords with the results in $\S 2.5$, where the maximum disturbance (shown in figure 3) also occurs at the steepest point of the shelf, which coincides there with the shelf-ocean boundary. The results here (figure 12) show that certain bay geometries support trapped modes (because of the wall effect) and 


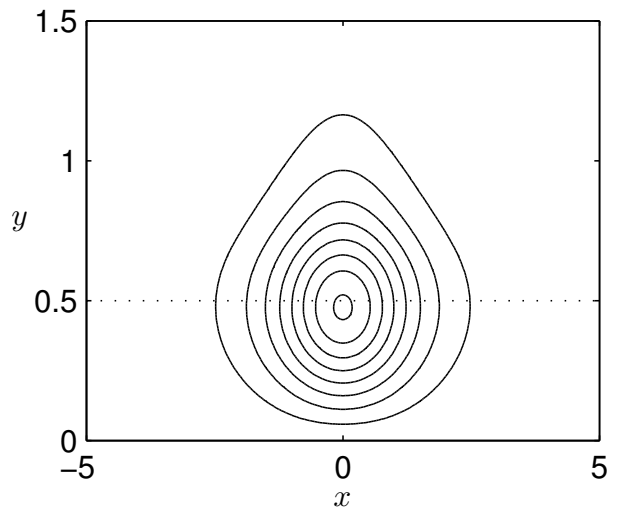

(a)

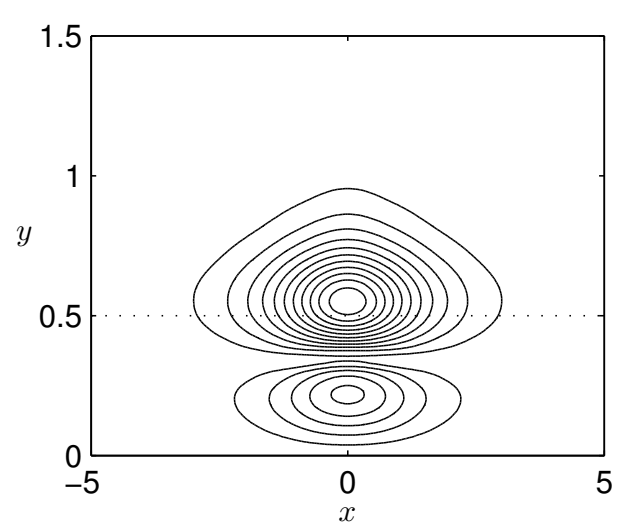

(b)

Figure 13. Contour plot of $|\psi|$ for (a) the mode $\omega_{1,1}$ and (b) the mode $\omega_{1,2}$ for the slope effect problem. The dotted line shows the position of the shelf break

the corresponding headland geometries do not. On the other hand, figure 11 shows an example where headlands support trapping and bays do not (because of the curvature effect). Similarly, figure 13 shows the existence of trapped modes along a valley due to the slope effect, while the trapping for the ridge geometry in $\S 2.5$ is due to the combination of the wall and the slope effect.

\section{Discussion}

We demonstrate the existence of trapped CSWs along straight coastlines featuring both alongshore and cross-shore depth variations. No rigorous proof of their existence is given, however, strong evidence is provided by accurate numerical solutions and from an asymptotic solution. Assuming that the scale of the along-shelf variations of the isobaths is large compared to the shelf width, we reduce our problem to finding (analytical) solutions of a second order ordinary differential equation in the cross-shelf direction. It is shown that in the case of a submerged ridge, when the depth is perturbed slightly, a trapped mode always exists, while in the case of a submerged valley, trapping is impossible. Direct comparison between the asymptotic method and the numerical method, shows that the results from the two methods are in agreement. We also show numerically, that trapped modes still exist when the shelf extends into the open ocean, thus removing the Neumann approximation taken at the shelf-ocean boundary and give an assessment of this approximation.

Previous results by JLP, Postnova and Craster (2008), and Kaoullas and Johnson (2010b), are extended by showing numerically, that trapped modes exist along curved coastlines with no restrictions to the curvature or requiring to approximate the boundary condition at the shelf-ocean boundary. In addition to the isobath curvature, two other geometric factors noted by Johnson and Kaoullas (2011), the wall and slope effect are also shown to be (individually or combined) necessary and sufficient for the existence of trapping. The underlying physics are also examined showing how each of these factors raises or lowers the cut-off frequency for propagating modes locally, at the topographic feature, compared to the cut-off frequency in the farfield (which remains unchanged), and thus allowing to identify features where trapping is physically expected. Since the numerical method does not require the governing equation to have constant coefficients 
these results were obtained for a wider class of topographies (rather than convex exponential or power-law cross-shelf profiles used extensively in the literature) and can also be applied by oceanographers to shelf geometries from observed topographic data, provided the data is approximated by a smooth interpolating function.

How far the frequencies of trapped modes lie above the cut-off frequency in the farfield, which determines how clearly differentiated these modes are from propagating modes, depends on how strongly the topography is perturbed in the trapping region. For order unity disturbances the frequency differences are order unity and this is when such modes would be likely to be identified in practice. In the possible example of trapped modes put forward in Stocker and Johnson (1991) the estuary was an order unity perturbation to shelf as was the lake end in Trösch (1986) and Johnson and Kaoullas (2011). For the weak perturbations of the asymptotic theory the length scale and size of the the topographic feature are inversely related and together determine how far the frequency of trapping lies above cut-off with small, long perturbations leading to trapping frequencies only slightly above cutoff (Johnson et al. 2011).

Relaxing the rigid-lid approximation thus allowing the ocean surface to be free introduces the super-inertial Poincarè waves and the free-surface (external) Kelvin wave which is present at all frequencies. If the external Rossby radius $R$ (LeBlond and Mysak 1978) is of order unity or less (i.e. shallow, very wide shelves or shelves near the equator) this approximation fails and we are dealing with a completely different mathematical problem. Nevertheless, for typical, mid-latitude shelves, due to the disparity in scales between the Kelvin wave and shelf waves any energy transfer will be negligible (Buchwald and Adams 1968; LeBlond and Mysak 1978).

Introducing stratification increases the wave frequency at all alongshore wavenumbers (Mysak 1980; Brink 1991). If the stratification is strong enough the group velocity is generally in the same direction as the phase velocity and the short waves carrying energy in the opposite direction of long waves, disappear and so trapping is impossible. However, for sufficiently weak stratified shelves, some subinertial waves remain evanescent (Huthnance 1978; Chapman 1983), and trapping is possible in a reduced range of frequencies. Further discussion and numerical examples of localised coastal trapped waves ( $\ell$ CTWs) are given in Rodney and Johnson (2012).

The authors thank the UK Engineering and Physical Sciences Research Council for supporting this work under Grant EP/ D058864/1.

\section{Appendix A}

Introduce points $z_{i, j}$ for $i=1, \ldots, N$ and $j=1, \ldots, M$, to give a grid of $N$ points in the $y$-direction and $M$ in the $x$-direction, let $u_{i, j}$ be values of the solution of $(2.26)$ at the points $z_{i, j}$ defined as

$$
u_{i, j}=\left(u_{1,1}, u_{2,1}, \cdots, u_{N, 1}, u_{1,2}, \cdots, u_{N, 2}, \cdots, u_{1, M}, \cdots, u_{N, M}\right)^{T},
$$

and define the differentiation matrices

$$
\frac{\partial^{n}}{\partial y^{n}}=\mathbf{I}_{M} \otimes\left(\frac{2}{b-a}\right)^{n} \hat{\mathbf{D}}_{y}^{n}, \quad \frac{\partial^{n}}{\partial x^{n}}=\left(\frac{2}{L}\right)^{n} \hat{\mathbf{D}}_{x}^{n} \otimes \mathbf{I}_{N},
$$

where $\hat{\mathbf{D}}_{x}^{n}$ and $\hat{\mathbf{D}}_{y}^{n}$ are the Chebyshev differentiation matrices as defined in Weideman and Reddy (2000), $\mathbf{I}_{M}$ is the $M \times M$ identity matrix, $n$ is the order of the derivative and $\otimes$ is the tensor product. In order to impose the Dirichlet boundary condition we need to remove the first or last row and column of the matrix $\hat{\mathbf{D}}^{n}$, depending on which end 
of domain is imposed. For the Neumann condition a Hermite interpolation approach is used given in Weideman and Reddy (2000). The discretised equation can be written as

$$
\omega^{2} \mathbf{A} \boldsymbol{u}+\omega \mathbf{B} \boldsymbol{u}+\mathbf{C} \boldsymbol{u}=0,
$$

where (for the symmetric case) $\mathbf{A}, \mathbf{B}$ and $\mathbf{C}$ are $(M-1)(N-2) \times(M-1)(N-2)$ matrices:

$$
\begin{aligned}
& \mathbf{A}=\mathbf{D}_{x}^{2}+\mathbf{D}_{y}^{2}-2 b\left(\mathbf{P}_{x} \mathbf{D}_{x}+\mathbf{P}_{y} \mathbf{D}_{y}\right), \\
& \mathbf{B}=2 b \mathrm{i}\left(\left(\mathbf{I}-\mathbf{P}_{y}\right) \mathbf{D}_{x}+\mathbf{P}_{x}\left(\mathbf{D}_{y}-b \mathbf{I}\right)\right), \\
& \mathbf{C}=b^{2}\left(2 \mathbf{P}_{y}-\mathbf{I}\right),
\end{aligned}
$$

the matrices $\mathbf{P}_{x}$ and $\mathbf{P}_{y}$ are the values of the functions

$$
p_{x}=-y(1-y) \alpha \epsilon^{3} \operatorname{sech}^{2}(\epsilon x) \tanh (\epsilon x), \quad p_{y}=1+(1-2 y) \alpha \epsilon^{2} \tanh (\epsilon x) / 2,
$$

at the points $z_{i, j}, \mathbf{I}$ is the $(M-1)(N-2) \times(M-1)(N-2)$ identity matrix and $\mathbf{D}^{n}$ are the differentiation matrices subject to the appropriate boundary conditions. System (A 2) can be transformed into a linear system by introducing the unknown vector $\boldsymbol{v}=\omega \boldsymbol{u}$ giving

$$
\begin{aligned}
\mathbf{C u}+\mathbf{B} \boldsymbol{v} & =-\omega \mathbf{v} \\
\boldsymbol{v} & =\omega \boldsymbol{u} .
\end{aligned}
$$

Since the frequencies of trapped modes lie above, but close to the cutoff frequency $\omega_{0}$, of the rectilinear shelf in the farfield, numerical efficiency can be further increased by introducing the shifted eigenvalue $\lambda$ as $\omega=\omega_{0}+\lambda$ which satisfies the standard eigenvalue problem

$$
\mathcal{F} \boldsymbol{w}=\lambda \boldsymbol{w},
$$

where $\mathcal{F}=\mathbf{G}^{-1} \mathbf{F}, \boldsymbol{w}=(\boldsymbol{u}, \boldsymbol{v})^{T}$ and

$$
\mathbf{F}=\left(\begin{array}{cc}
\mathbf{C} & \mathbf{B}+\omega_{0} \mathbf{A} \\
-\omega_{0} \mathbf{I} & \mathbf{I}
\end{array}\right), \quad \mathbf{G}=\left(\begin{array}{cc}
\mathbf{0} & -\mathbf{A} \\
\mathbf{I} & \mathbf{0}
\end{array}\right)
$$

\section{REFERENCES}

M. Abramowitz and I. A. Stegun. Handbook of Mathematical Functions with Formulas, Graphs, and Mathematical Tables. Dover, New York, tenth edition, 1964.

A. Aslanyan, L. Parnovski, and D. Vassiliev. Complex resonances in acoustic waveguides. Quart. J. Mech. Appl. Math., 53(3):429-447, 2000.

J. P. Boyd. Chebyshev and Fourier Spectral Methods. Dover, New York, 2000.

K. H. Brink. Coastal-trapped waves and wind-driven currents over the continental shelf. Annual Review of Fluid Mechanics, 23(1):389-412, 1991.

V. T. Buchwald and J. K. Adams. The propagation of continental shelf waves. Proc. R. Soc Lond. A, 305(1481), 1968.

D. C. Chapman. On the influence of stratification and continental shelf and slope topography on the dispersion of subinertial coastally trapped waves. J. Phys. Oceanogr., 13(9):1641-1652, 1983.

E. B. Davies and L. Parnovski. Trapped modes in acoustic waveguides. Quart. J. Mech. Appl. Math., 51(3):477-492, 1998.

J. Dittrich and J. Kriz. Curved planar quantum wires with dirichlet and neumann boundary conditions. J. Phys. A, 35(20):L269-L275, 2002.

P. Duclos and P. Exner. Curvature-induced bound states in quantum waveguides in two and three dimensions. Rev. Math. Phys., 7:73-102, 1995.

D. V. Evans, M. Levitin, and D. Vassiliev. Existence theorems for trapped modes. J. Fluid Mech., 261:21-31, 1994. 
P. Exner and P. Seba. Bound states in curved quantum waveguides. J. Math. Phys., 30(11): 2574-2580, 1989.

D. Gridin, R. V. Craster, and A. T. I. Adamou. Trapped modes in curved elastic plates (vol 461, pg 1181, 2005). Proc. R. Soc Lond. A, 461(2064):1181-1197, 2005.

B. V. Hamon. Spectrums of mean sea level at Sydney, Coff's Harbour, and Lord Howe Island. J. Geophys. Res., 67(13):5147-5155, 1962.

B. V. Hamon. Correction to the spectrums of mean sea level at Sydney, Coff's Harbour, and Lord Howe Island. J. Geophys. Res., 68(15):4635, 1963.

J. M. Huthnance. Coastal trapped waves - analysis and numerical-calculation by inverse iteration. J. Phys. Oceanogr., 8(1):74-92, 1978.

E. R. Johnson. Topographic waves in open domains. part 1. boundary conditions and frequency estimates. J. Fluid Mech., 200:69-76, 1989.

E. R. Johnson and G. Kaoullas. Bay-trapped low-frequency oscillations in lakes. Geophys. Astrophys. Fluid Dyn., 105(1):48 - 60, 2011.

E. R. Johnson, M. Levitin, and L. Parnovski. Existence of eigenvalues of a linear operator pencil in a curved waveguide-localized shelf waves on a curved coast. SIAM J. Math. Anal., 37 (5):1465-1481, 2006.

E. R. Johnson, J. Rodney, and G. Kaoullas. Trapped modes in coastal waveguides. (Wave Motion Sub Judice), 2011.

G. Kaoullas and E. R. Johnson. Fast accurate computation of shelf waves for arbitrary depth profiles. Continental Shelf Research, 30(7):833 - 836, 2010a.

G. Kaoullas and E. R. Johnson. Geographically localised shelf waves on curved coasts. Continental Shelf Research, 30(15):1753 - 1760, 2010b.

J. D. Kaplunov, G. A. Rogerson, and P. E. Tovstik. Localized vibration in elastic structures with slowly varying thickness. Quart. J. Mech. Appl. Math., 58(Part 4):645-664, 2005.

D. Krejčiřík and J. Kříž. On the Spectrum of Curved Planar Waveguides. Publ. Res. Inst. Math. Sci., 41(3):757-791, 2005.

L. D. Landau and E. M. Lifshitz. Quantum Mechanics Non-Relativistic Theory : Volume 3. Butterworth-Heinemann, Oxford, 1991.

P. H. LeBlond and L. A. Mysak. Waves in the ocean. Elsevier, Amsterdam, 1978.

L. A. Mysak. Recent advances in shelf wave dynamics. Rev. Geophys. Space Phys., 18(1): 211-241, 1980.

A. H. Nayfeh. Introduction to Perturbation Techniques. John Wiley, New York, 1993.

J. Postnova and R. V. Craster. Trapped modes in elastic plates, ocean and quantum waveguides. Wave Motion, 45(4):565-579, 2008.

A. R. Robinson. Continental shelf waves and the response to of sea level to weather systems. $J$. Geophys. Res., 69(2):367-368, 1964.

J. T. Rodney and E. R. Johnson. Localisation of coastal trapped waves by longshore variations in bottom topography. Continental Shelf Research, 32:130-137, 2012.

J. Shen and L.-L. Wang. Some Recent Advances on Spectral Methods for Unbounded Domains. Commun. Comput. Phys., 5(2-4):195-241, 2009.

T. Stocker and K. Hutter. One-dimensional models for topographic rossby waves in elongated basins on the f-plane. J. Fluid Mech., 170:435-459, 1986.

T. Stocker and K. Hutter. Topographic waves in rectangular basins. J. Fluid Mech., 185: 107-120, 1987.

T. Stocker and E. R. Johnson. Topographic waves in open domains. part 2. bay modes and resonances. J. Fluid Mech., 200:77-93, 1989.

T. Stocker and E. R. Johnson. The trapping and scattering of topographic waves by estuaries and headlands. J. Fluid Mech., 222:501-524, 1991.

G. Szegö. Orthogonal Polynomials, volume 23. American Mathematical Society Colloqium Publications, 4th edition, 1975.

T. Tang. The hermite spectral method for gaussian-type functions. SIAM J. Sci. Comput., 14 (3):594-606, 1993.

L. N. Trefethen. Spectral Methods in MATLAB. SIAM, 2000.

J. Trösch. Finite element calculation of topographic waves in lakes. In Proceedings of the 4 th International Conference on Applied Numerical Modeling (Tainan, Taiwan, 1984), Science 
and Technology Series 63, pages 307-311. H. M. Hsia, Y. L. Chou, S. Y. Wang, and S. J. Hsieh, eds., American Astronautical Society, 1986.

J. A. C. Weideman and S. C. Reddy. A MATLAB differentiation matrix suite. $A C M, 26(4)$ : 465-519, DEC 2000. 